\title{
Neuroinflammation in primary blast neurotrauma: time course and prevention by torso
}

\section{shielding}

\section{Leyan $\mathrm{Xu}^{\mathrm{a}}$}

${ }^{a}$ Division of Neuropathology, Department of Pathology, The Johns Hopkins University School of Medicine, Baltimore, Maryland, 21205, USA

Email: 1xu9@jhmi.edu

Michele L. Schaefer ${ }^{\mathrm{d}}$

National Security Technology, Johns Hopkins University Applied Physics Laboratory, Laurel, MD, 20723, USA

Email: mschae11@jhmi.edu

Raleigh M. Linville ${ }^{d}$

National Security Technology, Johns Hopkins University Applied Physics Laboratory, Laurel, MD, 20723, USA

Email: raleigh@bu.edu

Ayushi Aggarwal $^{\mathrm{d}}$

${ }^{\mathrm{a} D i v i s i o n}$ of Neuropathology, Department of Pathology, The Johns Hopkins University School of Medicine, Baltimore, Maryland, 21205, USA

Email:ayushi1@umbc.edu

Wangui Mbuguiro ${ }^{\mathrm{d}}$

National Security Technology, Johns Hopkins University Applied Physics Laboratory, Laurel, MD, 20723, USA

Email: wmbuguiro@gmail.com

Brock A. Wester ${ }^{\mathrm{d}}$

National Security Technology, Johns Hopkins University Applied Physics Laboratory, Laurel, MD, 20723, USA

Email:Brock.Wester@jhuapl.edu

Vassilis E. Koliatsos ${ }^{\mathrm{a}, \mathrm{b}, \mathrm{c}}$

${ }^{\mathrm{a}}$ Division of Neuropathology, Department of Pathology, ${ }^{\mathrm{d}}$ Department of Neurology, ${ }^{\mathrm{e}}$ Department of Psychiatry and Behavioral Sciences, The Johns Hopkins University School of Medicine, Baltimore, Maryland, 21205, USA

Email: koliat@jhmi.edu

Corresponding author: Leyan Xu

Division of Neuropathology, Department of Pathology, The Johns Hopkins University School of Medicine, 720 Rutland Ave, Ross research Building 558, Baltimore, Maryland, 21205, USA

Phone: 410-502-5191

Email: $\underline{\text { xu9@jhmi.edu }}$ 


\begin{abstract}
Mechanisms of primary blast injury caused by overpressure are not fully understood. In particular, the presence and time course of neuroinflammation are unknown and so are the signatures of reactive inflammatory cells, especially the neuroprotective versus injurious roles of microglia. In general, chronic microglial activation in the injured brain suggests a pro-degenerative role for these reactive cells. In this study, we investigated the temporal dynamics of microglial activation in the brain of mice exposed to mild-moderate blast in a shock tube. Because, in our previous work, we had found that torso shielding with rigid Plexiglas attenuates traumatic axonal injury in the brain, we also evaluated neuroinflammatory microglial responses in animals with torso protection at 7 days post blast injury. Because of the prominent involvement of the visual system in blast TBI in rodents, activated microglial cells were counted in the optic tract at various time points post-injury with stereological methods. Cell counts (activated microglial cell densities) from subjects exposed to blast TBI were compared with counts from corresponding sham animals. We found that mild-moderate blast injury causes focal activation of microglia in certain white matter tracts, including the visual pathway. In the optic tract, the density of activated microglial profiles gradually intensified from 3 to 15 days post-injury and then became attenuated at 30 days. Torso protection significantly reduced microglial activation at 7 days.

These findings shed light into mechanisms of primary blast neurotrauma and may suggest novel diagnostic and monitoring methods for patients. They leave open the question of whether microglial activation post blast is protective or detrimental, although response is time limited. Finally, our findings confirm the protective role of torso shielding and stress the importance of improved or optimized body gear for warfighters or other individuals at risk for blast exposure.
\end{abstract}

\title{
Key words:
}

Primary blast injury, traumatic axonal injury, neurotrauma, microglia, optic tract

\section{Abbreviations:}


CV, cresyl violet; DAB, 3,3'-diaminobenzidine; DAPI, 4', 6-diamidino-2-phenylindole; IHC, immunohistochemistry; IL-1ra, IL-1 receptor antagonist; NGF, nerve growth factor; ROI, regions of interest; TAI, traumatic axonal injury; TBI, traumatic brain injury; TGF $\beta$, transforming growth factor $\beta$

\section{Introduction}

Blast-induced neurotrauma is a common cause of traumatic brain injury (TBI) and the "signature injury" in recent conflicts in Iraq and Afghanistan. Blast neurotrauma is complex and its mechanisms are not fully understood, especially for primary blast injury that is the direct result of overpressure wave (Cernak et al., 1999;Ling et al., 2009;Rosenfeld et al., 2013). Most cases of blast neurotrauma are mild (concussion) and are not associated with gross damage to brain parenchyma (Rosenfeld et al., 2013;Chapman and Diaz-Arrastia, 2014;Bell et al., 2009).

We have recently developed and characterized a mouse model of primary blast neurotrauma using a specially designed shock-tube (Cernak et al., 2011;Koliatsos et al., 2011). This model generates neurological morbidity and mortality depending on shock-wave intensity (Cernak et al., 2011;Koliatsos et al., 2011). Under mildmoderate exposure conditions, subjects show traumatic axonal injury (TAI) in select tracts, especially optic and corticospinal, and develop mild cognitive/behavioral impairments (Koliatsos et al., 2011). One notable finding is that torso shielding significantly reduces TAI and mitigates behavioral defects, but head shielding does not offer any protection (Cernak et al., 2011;Koliatsos et al., 2011).

Neuroinflammation occurs frequently after blunt TBI, including concussion, and may contribute to secondary injury (Kumar and Loane, 2012;Morganti-Kossmann et al., 2002). Much less is known about neuroinflammation in blast neurotrauma. Neuroinflammation is featured by activation of microglia, i.e. mesodermally derived immunocompetent cells that exist in a resting state until they sense an injury signal and then become activated. A component of this activation is the formation of cells with classical macrophage properties that may further damage neural parenchyma (Morganti-Kossmann et al., 2002;Finnie, 2013;Acosta et al., 2013). Blocking microglial activation with minocycline may ameliorate neuropathology and functional or behavioral impairments in rodents subjected to TBI (Siopi et al., 2012;Homsi et al., 2010). Activated 
microglia can persist for long periods of time, a pattern indicating chronic neuroinflammation that may further damage the brain (Gentleman et al., 2004;Johnson et al., 2013).

In the present study, we investigated the temporal dynamics of neuroinflammation in the brain of mice exposed to mild-moderate blast in our established shock-tube model (Cernak et al., 2011;Koliatsos et al., 2011). Neuroinflammation was assessed by the presence of reactive microglia in injured brain tissues after mild-moderate blast, with and without torso protection. Although reactive astrocytes are part of the neuroinflammatory response to injury, these cells play complex roles that are difficult to dissect within the scope of this study (Ridet et al., 1997;Sofroniew, 2005;Anderson et al., 2014;Brenner, 2014;Pekny and Pekna, 2014;Pekny et al., 2014). The optic tract, one of the primary sites of TAI after blast (Koliatsos et al., 2011) was used here for purpose of quantitative assessment. Our findings are consistent with the view that neuroinflammation in primary blast neurotrauma tends to resolve one month after injury and confirm our previous findings that torso protection prevents key aspects of blast neurotrauma. These findings may assist the development of imaging strategies to monitor progression or resolution of primary blast neurotrauma and strategies to prevent secondary brain injury after blast with optimal armor protection or treat blast-induced neuroinflammation.

\section{Materials and methods}

\section{Experimental Subjects and shock tube set-up}

The subjects of these experiments were 7-8 week old male C57BL6/J mice (Charles River Laboratories, Wilmington, MA). All animal care, operative and post-operative procedures were approved by the Animal Care and Use Committee of the Johns Hopkins Medical Institutions.

Animals were subjected to blast neurotrauma via shockwave generated in a multi-chamber shock tube as described (Cernak et al., 2011;Koliatsos et al., 2011). Briefly, subjects were anesthetized with a gas mixture (isoflurane: nitrous oxide: oxygen 1:66:33) and placed in a multi-chamber shock tube consisting of two interlocking pipe compartments (a driver and a driven compartment) separated by an intervening Kapton- 
polyethylene diaphragm (Cernak et al., 2011). Anesthetized animals were fixed on a wire-mesh holder by tethering the four limps with cotton threads and holder was positioned at the open end of the shock tube facing the shockwave front. To minimize movement of head, the upper frontal teeth were also tethered on the mesh by a cotton thread encircling the snout. Shockwave was generated by release of compressed helium in the driver compartment. Shockwave was adjusted to generate a membrane rupture pressure of 30 psig corresponding to mild-moderate blast injury (Cernak et al., 2011;Koliatsos et al., 2011).

To evaluate the role of body shielding in neuropathologies associated with blast injury, one group of subjects was fitted with a Plexiglas shield covering the chest and abdomen as described (Cernak et al., 2011;Koliatsos et al., 2011). Control mice were given sham lesions as previously described (Cernak et al., 2011; Koliatsos et al., 2011). These subjects were mounted to the side of the shock tube, thus they were exposed to the sound but not the shockwave itself.

Animals were allowed to survive for $1,3,7,14$ and 30 days (average $n=5$ for each time point). Injured animals with torso protection were only allowed to survive for 7 days. All animals were euthanized with perfusion fixation and tissues were processed as described in next section.

\section{Histology, general histochemistry and immunohistochemistry}

At the appropriate times, animals were perfused with $4 \%$ freshly depolymerized, neutral-buffered paraformaldehyde. Brains were removed from skulls and immersed in the same fixative overnight at $4{ }^{\circ} \mathrm{C}$. Tissues were cryoprotected and stored at $-80{ }^{\circ} \mathrm{C}$ for further processing. Coronal brain sections $(40 \mu \mathrm{m})$ were prepared in series for cresyl violet (CV), immunohistochemistry (IHC) and dual-label fluorescent IHC for markers of neuroinflammatory cells (the universal microglial marker IBA1 and the scavenger receptor/lysosomal membrane protein CD68 as a macrophage marker).

Immunoperoxidase-DAB based IHC was performed as previously described (Koliatsos et al., 2011; Xu et al., 2009). Briefly, sections were first incubated in the primary antibody overnight at $4{ }^{\circ} \mathrm{C}$ and then in biotinconjugated secondary antibody (1:200; Jackson ImmunoResearch, West Grove, PA). Then sections were 
incubated in a solution of avidin and biotinylated HRP complex (VECTASTAIN Elite ABC Kit, VECTOR laboratories Inc., Burlingame, CA), then developed in 3,3'-diaminobenzidine (DAB). Some sections were counter-stained with CV. For fluorescent IHC, sections were incubated in secondary antibodies conjugated with Cy3 or Cy2 (1:200; Jackson ImmunoResearch, West Grove, PA) for 2-4 hours at room temperature and then counterstained with the fluorescent DNA dye 4', 6-diamidino-2-phenylindole (DAPI) and coverslipped with DPX mounting media. Primary antibodies included: rat anti-CD68 (1:500, ABD Serotec, Raleigh, NC), rabbit anti-IBA-1 (1:500; Dako, Carpinteria, CA), mouse anti-KV1.3 voltage-gated potassium channel 1.3 (Kv1.3) (1:1000, NeuroMab Davis, CA). The latter epitope has been associated with presumably neurotoxic small ramified microglia (Yamada and Jinno, 2013). In the present study, no distinct above-background Kv1.3 (+) immunoreactivity was found in the optic tract 14 and 30 days postinjury using the same antibody as Yamada and Jino and no further comment will be provided in the Results.

Labeled sections were studied with a Zeiss Axiophot microscope and images were captured with a Spot RT Slider digital camera (Diagnostic Instruments, Inc., Sterling Heights, MI). Confocal microscopy images were captured with pinhole set at $0.8 \mu \mathrm{m}$. Three-dimensional reconstruction by Z-stack scanning through regions of interest (ROI) was acquired with LSM software. Adobe Photoshop 7.0 software (Adobe Systems, San Jose, CA) was used for image processing.

\section{Microglial profile counts in optic tract}

Numbers of activated microglial profiles were counted in serial, systematically and randomly sampled coronal sections based on the optical fractionator concept as described (Yan et al., 2007). Counts were performed with an Axioplan microscope (Zeiss, www.zeiss.de) equipped with a motorized-stage and Stereo Investigator software version 10.01 (MicroBrightField, www.mbfbioscience.com). Every $24^{\text {th }}$ of serial sections through the optic tract (from optic chiasm to cerebral peduncle) was subjected to stereological analysis. Contours were outlined at 5x and cells were counted using a 40x objective by a blinded investigator. A $125 \times 125 \mu$ m counting frame was used with a $125 \mu \mathrm{m} \times 125 \mu \mathrm{m}$ grid in the outlined area. Counting depth (optical dissector height) 
was $8-13 \mu \mathrm{m}$ depending on average section thickness. A guard volume of $1.0 \mu \mathrm{m}$ was used to avoid sectioning artifacts such as lost caps and uneven section surfaces. Density of microglia profiles was calculated by dividing estimated counts of profiles generated automatically from software with the estimated volume of the optic tract.

For CD68 (+) microglia, single cells and clusters of cells were counted separately. For IBA-1 (+) microglia, because of some difficulties in differentiating among various types of activated IBA-1 (+) microglial profiles, e.g. hypertrophied versus bushy/amoeboid and bushy/ amoeboid versus densely packed microglial cell clusters, all activated microglial profiles were counted together as a single cytological entity. Although such an approach may reduce statistical significance when we measure the effect of blast, it is more accurate than the densitometry method in which results can be distorted by many factors, such as numbers of resting microglial cells, extent of process retraction during activation and variation in background among individual samples.

\section{Statistical Analysis}

Variance in microglial profile counts between injured and corresponding sham groups was assessed by twotailed student $t$-test. Variance in microglial profile counts among sham, injured (unprotected) and injured, torso-protected groups was assessed by one-way ANOVA followed by Tukey post hoc testing.

\section{Results}

\section{General microglial responses after mild-moderate blast injury}

Blast injury caused microglial activation in various white matter regions at all time points studied. These regions included the optic tract, superior colliculus, lateral geniculate nucleus, pyramidal tract, fornix, lateral lemniscus, arbor vitae in cerebellum, and the corpus callosum. IBA-1(+) deramified microglia overlapped substantially with CD68 (+) microglia in these areas, a pattern confirming the regional pattern of axonal injury in this model. However, location of microglial activation varied among individual cases and differed based on time post-injury. The optic tract and superior colliculus were the regions most frequently involved, a pattern 
suggesting that mild-moderate blast injury in mice has a propensity to affect the visual system. In other regions, microglial activation was quite variable, a condition that made it very difficult to count either IBA1(+) or CD68 (+) activated microglia at these sites. Therefore, to explore the temporal dynamics of microglial activation and the role of body shielding in preventing blast neurotrauma, the optic tract was selected as target of investigation.

Microglial activation was featured by retraction of processes (deramification) and enlargement of the cell body (Figure 1). When labeled with IBA-1 antibodies, activated microglial cells presented with a variety of cytologies, including "hypertrophic" and "bushy" or "amoeboid" profiles (Figure 1B-C). Cresyl violet counterstaining demonstrated that some of the largest IBA-1 (+) bushy microglia were actually clusters of 2-5 deramified microglial cells (Figure 1D, Figure 2). In contrast to IBA-1 IHC that labeled both ramified (resting) and deramified (activated) microglia, CD68 IHC appeared to only label deramified microglia (Figure 1E). As in the case of IBA-1 IHC, CD68-immunoreactive microglial cells frequently formed clusters that were easy to misconstrue as single large cells with conventional microscopy (Figure 1F), although confocal microscopy revealed nicely their cytological complexity as well as the granular nature of CD68 immunoreactivity in individual cells (Figure 2).

\section{Time course of changes in IBA-1(+) microglial profiles in optic tract after blast injury}

As mentioned in the previous section, mild-moderate blast injury caused extensive microglial activation in the optic tract (Figure 3A-B). The density of IBA-1 (+) activated microglial profiles in the optic tract gradually increased from 3 to 7 days post-injury and then became attenuated between 15 and 30 days (Figure 3C). Significant differences in density of IBA-1 (+) activated microglia between injury and sham groups were found at 7 and 15 days (Figure 3C). The density of the entire population of IBA-1(+) microglial profiles showed a similar time course pattern as the population of activated profiles (Figure 3D). There was also a significant increase of total microglial profiles at 7 and 14 days post-injury, suggesting that at least a portion of the microglial population at later time points may have originated from proliferating precursors. 
Because activated microglial cells have few CD68-immunoreactive branches, it is relatively easy to separate individual from clustered CD68 (+) activated microglial profiles. Thus, quantitation of CD68 (+) activated microglia in the optic tract was performed separately for single and clustered activated cells.

In a fashion similar to the results of IBA-1 IHC, blast injury increased the density of CD68 (+) activated microglial profiles in the optic tract (Figure 4A-B). As in the case of IBA-1 IHC, stereological counts show that the density of CD68 (+) microglia (both single and clustered cell profiles) in the optic tract gradually increased from 3 to 7 days post-injury and then became attenuated by 30 days (Figure 4C-D). Significant differences in densities of single CD68 (+) activated profiles between injured and sham groups were noted at 3 days and 7 days, whereas significant differences in CD68 (+) microglial clusters were noted at 7 and 14 days after injury (Figure 4C-D).

\section{Prevention of blast-induced neuroinflammation with torso shielding}

Densities of IBA-1 (+) and CD68 (+) activated microglial profiles (as single cells and clusters) in the optic tract were significantly lower in animals protected by torso shielding at 7 days post-injury (Figure 5). Densities of activated IBA-1 (+) or CD68 (+) profiles were not significantly different between sham and injured, torso-protected animals (Figure 5). These results indicate that, at least in the case of optic tract, torso protection can reduce or eliminate neuroinflammation after mild-moderate blast injury.

\section{Discussion}

The present study expands on our previous work with single primary blast injury in mice where we demonstrated multifocal TAI in the optic tract, corticospinal system, and cerebellum (Koliatsos et al., 2011). Our findings indicate that, in addition to TAI, microglial activation is also an important aspect of blast-induced neuropathologies and that such a neuroinflammatory response occurs in the same locations as axonal injury. Emphasis on the optic tract is justified by the fact that TAI in this area is marked and concentrated (Koliatsos et al., 2011), and intensity in microglial response is more consistent compared to brain areas with more “diluted" TAI. 
Our findings show that, after mild-moderate blast injury, there is progressive increase of IBA1 (+) or CD68 (+) activated microglial cells in the injured optic tract from 3 to 15 days and return to baseline at 30 days postinjury. To the extent the focal neuroinflammation observed in our study reflects the presence of TAI, this temporal course suggests that axonal injury may cease to progress after 30 days and injured axons have either recovered or have been cleaned up by activated microglia. Naturally, we cannot rule out the possibility that severe blast neurotrauma or repetitive mild-moderate injury may cause chronic degeneration (Aungst et al., 2014). Although one study indicated that a single blast injury event can cause CTE-like tauopathy (Goldstein et al., 2012), we (Xu et al., 2014) and others (Mouzon et al., 2014) have failed to show tau hyperphosphorylation or aggregation in wild-type animals after single or repetitive concussive injury. Also, we were not able to see tau hyperphosphorylation in our material of blast-exposed mice from a previous study (Koliatsos et al., 2011). On the other hand, single or repetitive mild TBI exacerbates tauopathy in transgenic mice with aggregation-prone human tau or normal human tau but under abnormal ratio of 3R to $4 \mathrm{R}$ (Xu et al., 2015). Together, our interpretation of the literature in light of our present findings is consistent with the view that, at this point, there is little evidence to suggest that single mild-moderate TBI, including primary blast neurotrauma, can lead to chronic neurodegenerative disease in genetically normal subjects. One key difference between our study and that of Goldstein et al (Goldstein et al., 2012) is the degree to which head movement was allowed. In our study, the head was tethered onto mesh to minimize the head movement during the blast; thus, acceleration/deceleration (tertiary) injury was prevented (Gullotti et al., 2014). Axonal injury and neuroinflammation in our model were mainly caused by the primary effect of blast wave.

Although some degree of microglial activation has been observed across the board in human TBI and animal models, and as such it may serve as a TBI biomarker (Hernandez-Ontiveros et al., 2013), reporting on various cytologies and phenotypes of activated microglial cells has been variable. For example, clusters of deramified microglial cells have been described in some (Geddes et al., 1997;Dissing-Olesen et al., 2007;Bachstetter et al., 2013;Shitaka et al., 2011) but not in other studies (Homsi et al., 2010;Cao et al., 2012;Kelley et al., 2007;Csuka et al., 2000), Cluster formation can be either a grouping phenomenon as individual activated microglia are quickly recruited to the injury site (Dibaj et al., 2011) or a local proliferative phenomenon, based 
on evidence that such cells may be positive for BrDU (Dissing-Olesen et al., 2007). In the present study, microglial clusters are also CD68 (+). Because CD68 is a marker for activated microglial cells, it appears that clustered cells are activated. Because microglia activation is a dynamic process, and the distinction between hypertrophied and bushy/amoeboid is not always reliable, we avoided further dissection between hypertrophied and bushy/amoeboid microglial cells and classified them together as activated microglial cells.

Based on general patterns of response of peripheral macrophages, activated microglia has been proposed to play detrimental as well as beneficial roles after TBI (Morganti-Kossmann et al., 2002). Such responses include classical activation into M1 killer cells that secrete high levels of IL-12 and low levels of IL-10, and M2 cells with an inverse IL-12/IL-10 secretion profile involved in tissue repair and suppressing inflammation (Stence et al., 2001;Suzumura et al., 1991;Liao et al., 2012; Cherry et al., 2014;Loane and Byrnes, 2010). However, due to overlapping expression of the previous markers and a dynamic conversion from one to another under various injury conditions and over time, the mechanisms of M1 and M2 microglial cells in TBI are not entirely clear. The weeks-long microglial activation after mild-moderate blast TBI may or may not be detrimental and its exact nature needs to be clarified further with additional studies. The apparent increase in the total number of IBA1 (+) profiles 7 and 14 days post blast may suggest a proliferative event in the second and third weeks, but such an assertion needs to be experimentally tested with BrdU or related microglial lineage studies.

Our findings show that shielding of the thoracic and abdominal cavities with a rigid Plexiglas case ameliorates neuroinflammation in the brain due to primary blast. These findings are consistent with the fact that the exact same shield also prevents TAI caused by primary mild-moderate blast (Koliatsos et al., 2011). One reason may be that torso shielding prevents TAI in the optic tract and that this, in turn, prevents microglial activation because of a lack of injury signal (Ryu et al., 2014). Another reason may be related to the possible role of spleen as an organ that modulates neuroinflammation (Ajmo, Jr. et al., 2008). Modulating immune responses through spleen, such as reducing IL-1 $\beta$, TNF- $\alpha$, and IL-6 contents, improves outcomes of neural injury (Acosta et al., 2015;Tajiri et al., 2014;Koliatsos et al., 2015;Li et al., 2011). Torso shielding can certainly protect the spleen from blast injury and this effect may attenuate microglial activation reported here. Together, 
these data support the view that interventions that prevent mechanical forces of the blast from impacting body cavities have important neuroprotective effects and that optimization of body gear worn by warfighters may substantially reduce primary blast neurotrauma.

There is an apparent discrepancy in cell counts between IBA-1 (+) activated microglia and CD68 (+) profiles (single cells plus clusters): at every time point studied, total CD68 (+) profiles are more numerous than IBA1(+) cells with activated cytologies. Two possible explanations are that CD68 antibodies also label nonactivated cells or that cell counts based on activated IBA1 (+) profiles tend to miss a significant number of activated cells, some of which may correspond to the small ramified cell of Yamada and Jinno (Yamada and Jinno, 2013). Of the two possibilities, the latter is more likely and raises concerns that the use of cytology as the sole method to determine activation status of microglia may not be always reliable.

If neuroinflammation is an important consequence of single mild-moderate blast event, then imaging neuroinflammation may serve as a practical and effective way of diagnosing primary blast neurotrauma, especially in the absence of direct ways to visualize traumatic axonal injury. The recent advent of PET ligands binding to translocator protein such as $\left[{ }^{11} \mathrm{C}\right](\mathrm{R})-\mathrm{PK} 11195$ and (18)F-GE-180 can be deployed to localize areas of injury and help monitor TBI progression and outcomes in living human subjects (Banati, 2002;Dickens et al., 2014;Coughlin et al., 2015).

In summary, our findings suggest that single mild-moderate blast exposure can cause focal neuroinflammation in areas also featured by TAI. Activated microglial cells are comprised of hypertrophic cells and cell clusters. Neuroinflammation abates by a month after injury. The role and type of microglia in this type of neuroinflammation, especially with respect to neurotoxic versus neuroprotective phenotypes, needs to be ascertained. These results shed light into mechanisms of primary blast neurotrauma and may suggest novel diagnostic and monitoring methods for the clinic. More importantly, our study confirms the protective role of torso shielding and stresses the importance of optimized body gear for warfighters and other individuals at risk for blast exposure.

\section{Acknowledgement}


This work was supported by DOD DM102465 (Cernak, PI, Koliatsos, subcontract PI) and also by Maryland TEDCO (2011-MSCRF-11 0067-00; 110902).

\section{Figures}

\section{Figure 1.}

Profiles of microglia at resting (A) and activated (B-F) status in the optic tract post blast injury. In A, IBA-1 (+) microglia have a ramified cytology, whereas in B and C they appear as hypertrophic or bushy/ amoeboid cells, respectively. Amoeboid microglial cells can group together to form clusters of 2-5 cells (D). Activated CD68 (+) microglia presents as either single (E) or clustered (F) cells. Scale bar: A-F, $10 \mu \mathrm{m}$.

\section{Figure 2.}

Confocal microscopic analysis of clusters of activated microglial cells in the lower optic tract post-blast injury. Preparations were stained with dual-fluorescent IHC for IBA-1 (green) and CD68 (red). Panels depict 3-5 densely packed IBA-1 (+) microglial cells expressing granular CD68 immunoreactivity (arrows) (3 cells in panel A and 5 cells in panel B). Colocalization of IBA-1 and CD68 signals is confirmed on the $\mathrm{x}$ and y planes at the indicated cross-sectional planes (arrowheads). A solitary CD68 (+) microglial cell is indicated with an asterisk in the left panel. Scale bars: $10 \mu \mathrm{m}$.

\section{Figure 3.}

Activation of IBA-1 (+) microglia in the optic tract post blast injury. The IHC figures show that activated profiles of IBA-1 (+) microglia are significantly higher in the injured optic tract at 7 days (A-B). Stereological counts of activated and total IBA-1 (+) microglial profiles are shown in (C) and (D). Data were analyzed with student's t-test. $* \mathrm{p}<0.05$. Scale bars: A-B, $50 \mu \mathrm{m}$ 


\section{Figure 4.}

Increase of CD68 (+) single activated and clustered microglial cells in the optic tract post-blast injury. IHC preparations show that activated CD68 (+) microglia (as single cells or clusters) is significantly increased in the injured optic tract at 7 days (A-B). Stereological counts of activated CD68 (+) single and clustered microglial profiles are shown in (C) and (D), respectively. Data were analyzed with student's t-test. * $\mathrm{p}<0.05$. Scale bars: A-B, $50 \mu \mathrm{m}$

\section{Figure 5.}

Stereological counts of activated microglial profiles in the optic tract with and without torso protection postblast injury. Bar graphs represent counts of activated IBA-1 (+) microglial profiles (A), CD68 (+) single microglial profiles (B), and clusters of CD68 (+) profiles (C) in the optic tract 7 days post-blast injury under sham, torso-protected (TP) and non-protected (No-TP) conditions. Data were analyzed with one-way ANOVA followed by Tukey post hoc testing. * $\mathrm{p}<0.05$.

\section{Reference List}

1. Acosta SA, Tajiri N, Hoover J, Kaneko Y, Borlongan CV (2015) Intravenous Bone Marrow Stem Cell Grafts Preferentially Migrate to Spleen and Abrogate Chronic Inflammation in Stroke. Stroke.

2. Acosta SA, Tajiri N, Shinozuka K, Ishikawa H, Grimmig B, Diamond DM, Sanberg PR, Bickford PC, Kaneko $Y$, Borlongan CV (2013) Long-term upregulation of inflammation and suppression of cell proliferation in the brain of adult rats exposed to traumatic brain injury using the controlled cortical impact model. PLOS ONE 8: e53376.

3. Ajmo CT, Jr., Vernon DO, Collier L, Hall AA, Garbuzova-Davis S, Willing A, Pennypacker KR (2008) The spleen contributes to stroke-induced neurodegeneration. J Neurosci Res 86: 2227-2234.

4. Anderson MA, Ao Y, Sofroniew MV (2014) Heterogeneity of reactive astrocytes. Neurosci Lett 565: 2329.

5. Aungst SL, Kabadi SV, Thompson SM, Stoica BA, Faden Al (2014) Repeated mild traumatic brain injury causes chronic neuroinflammation, changes in hippocampal synaptic plasticity, and associated cognitive deficits. J Cereb Blood Flow Metab 34: 1223-1232.

6. Bachstetter AD, Rowe RK, Kaneko M, Goulding D, Lifshitz J, Van Eldik L (2013) The p38alpha MAPK regulates microglial responsiveness to diffuse traumatic brain injury. J Neurosci 33: 6143-6153. 
7. Banati RB (2002) Visualising microglial activation in vivo. GLIA 40: 206-217.

8. Bell RS, Vo AH, Neal CJ, Tigno J, Roberts R, Mossop C, Dunne JR, Armonda RA (2009) Military traumatic brain and spinal column injury: a 5-year study of the impact blast and other military grade weaponry on the central nervous system. J Trauma 66: S104-S111.

9. Brenner M (2014) Role of GFAP in CNS injuries. Neurosci Lett 565: 7-13.

10. Cao T, Thomas TC, Ziebell JM, Pauly JR, Lifshitz J (2012) Morphological and genetic activation of microglia after diffuse traumatic brain injury in the rat. Neuroscience 225: 65-75.

11. Cernak I, Merkle AC, Koliatsos VE, Bilik JM, Luong QT, Mahota TM, Xu LY, Slack N, Windle D, Ahmed FA (2011) The pathobiology of blast injuries and blast-induced neurotrauma as identified using a new experimental model of injury in mice. Neurobiology of Disease 41: 538-551.

12. Cernak I, Savic J, Ignjatovic D, Jevtic M (1999) Blast injury from explosive munitions. Journal of TraumaInjury Infection and Critical Care 47: 96-103.

13. Chapman JC, Diaz-Arrastia R (2014) Military traumatic brain injury: a review. Alzheimers Dement 10 : S97-104.

14. Cherry JD, Olschowka JA, O'Banion MK (2014) Neuroinflammation and M2 microglia: the good, the bad, and the inflamed. J Neuroinflammation 11: 98.

15. Coughlin JM, Wang Y, Munro CA, Ma S, Yue C, Chen S, Airan R, Kim PK, Adams AV, Garcia C, Higgs C, Sair HI, Sawa A, Smith G, Lyketsos CG, Caffo B, Kassiou M, Guilarte TR, Pomper MG (2015)

Neuroinflammation and brain atrophy in former NFL players: An in vivo multimodal imaging pilot study. Neurobiol Dis 74: 58-65.

16. Csuka E, Hans VH, Ammann E, Trentz O, Kossmann T, Morganti-Kossmann MC (2000) Cell activation and inflammatory response following traumatic axonal injury in the rat. Neuroreport 11: 2587-2590.

17. Dibaj P, Steffens H, Zschuntzsch J, Kirchhoff F, Schomburg ED, Neusch C (2011) In vivo imaging reveals rapid morphological reactions of astrocytes towards focal lesions in an ALS mouse model. Neurosci Lett 497: 148-151.

18. Dickens AM, Vainio S, Marjamaki P, Johansson J, Lehtiniemi P, Rokka J, Rinne J, Solin O, HaaparantaSolin M, Jones PA, Trigg W, Anthony DC, Airas L (2014) Detection of microglial activation in an acute model of neuroinflammation using PET and radiotracers 11C-(R)-PK11195 and 18F-GE-180. J Nucl Med 55: 466-472.

19. Dissing-Olesen L, Ladeby R, Nielsen HH, Toft-Hansen H, Dalmau I, Finsen B (2007) Axonal lesion-induced microglial proliferation and microglial cluster formation in the mouse. Neuroscience 149: 112-122.

20. Finnie JW (2013) Neuroinflammation: beneficial and detrimental effects after traumatic brain injury. Inflammopharmacology 21: 309-320.

21. Geddes JF, Vowles GH, Beer TW, Ellison DW (1997) The diagnosis of diffuse axonal injury: implications for forensic practice. Neuropathol Appl Neurobiol 23: 339-347. 
22. Gentleman SM, Leclercq PD, Moyes L, Graham DI, Smith C, Griffin WS, Nicoll JA (2004) Long-term intracerebral inflammatory response after traumatic brain injury. Forensic Sci Int 146: 97-104.

23. Goldstein LE, Fisher AM, Tagge CA, Zhang XL, Velisek L, Sullivan JA, Upreti C, Kracht JM, Ericsson $M$, Wojnarowicz MW, Goletiani CJ, Maglakelidze GM, Casey N, Moncaster JA, Minaeva O, Moir RD, Nowinski CJ, Stern RA, Cantu RC, Geiling J, Blusztajn JK, Wolozin BL, Ikezu T, Stein TD, Budson AE, Kowall NW, Chargin D, Sharon A, Saman S, Hall GF, Moss WC, Cleveland RO, Tanzi RE, Stanton PK, McKee AC (2012) Chronic traumatic encephalopathy in blast-exposed military veterans and a blast neurotrauma mouse model. Sci Transl Med 4: 134ra60.

24. Gullotti DM, Beamer M, Panzer MB, Chen YC, Patel TP, Yu A, Jaumard N, Winkelstein B, Bass CR, Morrison B, Meaney DF (2014) Significant head accelerations can influence immediate neurological impairments in a murine model of blast-induced traumatic brain injury. J Biomech Eng 136: 091004.

25. Hernandez-Ontiveros DG, Tajiri N, Acosta S, Giunta B, Tan J, Borlongan CV (2013) Microglia activation as a biomarker for traumatic brain injury. Front Neurol 4: 30 .

26. Homsi S, Piaggio T, Croci N, Noble F, Plotkine M, Marchand-Leroux C, Jafarian-Tehrani M (2010) Blockade of acute microglial activation by minocycline promotes neuroprotection and reduces locomotor hyperactivity after closed head injury in mice: a twelve-week follow-up study. J Neurotrauma 27: 911-921.

27. Johnson VE, Stewart JE, Begbie FD, Trojanowski JQ, Smith DH, Stewart W (2013) Inflammation and white matter degeneration persist for years after a single traumatic brain injury. Brain 136: 28-42.

28. Kelley BJ, Lifshitz J, Povlishock JT (2007) Neuroinflammatory responses after experimental diffuse traumatic brain injury. J Neuropathol Exp Neurol 66: 989-1001.

29. Koliatsos VE, Cernak I, Xu L, Song Y, Savonenko A, Crain BJ, Eberhart CG, Frangakis CE, Melnikova T, Kim $H$, Lee $D$ (2011) A mouse model of blast injury to brain: initial pathological, neuropathological, and behavioral characterization. J Neuropathol Exp Neurol 70: 399-416.

30. Koliatsos VE, Xu L, Cummings BJ (2015) Stem cell therapies for traumatic brain injury. Regen Med.

31. Kumar A, Loane DJ (2012) Neuroinflammation after traumatic brain injury: opportunities for therapeutic intervention. Brain Behav Immun 26: 1191-1201.

32. Li M, Li F, Luo C, Shan Y, Zhang L, Qian Z, Zhu G, Lin J, Feng H (2011) Immediate splenectomy decreases mortality and improves cognitive function of rats after severe traumatic brain injury. J Trauma 71: 141147.

33. Liao B, Zhao W, Beers DR, Henkel JS, Appel SH (2012) Transformation from a neuroprotective to a neurotoxic microglial phenotype in a mouse model of ALS. Exp Neurol 237: 147-152.

34. Ling G, Bandak F, Armonda R, Grant G, Ecklund J (2009) Explosive Blast Neurotrauma. Journal of Neurotrauma 26: 815-825.

35. Loane DJ, Byrnes KR (2010) Role of microglia in neurotrauma. Neurotherapeutics 7: 366-377.

36. Morganti-Kossmann MC, Rancan M, Stahel PF, Kossmann T (2002) Inflammatory response in acute traumatic brain injury: a double-edged sword. Curr Opin Crit Care 8: 101-105. 
37. Mouzon BC, Bachmeier C, Ferro A, Ojo JO, Crynen G, Acker CM, Davies P, Mullan M, Stewart W, Crawford $F$ (2014) Chronic neuropathological and neurobehavioral changes in a repetitive mild traumatic brain injury model. Ann Neurol 75: 241-254.

38. Pekny M, Pekna M (2014) Astrocyte reactivity and reactive astrogliosis: costs and benefits. Physiol Rev 94: 1077-1098.

39. Pekny M, Wilhelmsson U, Pekna M (2014) The dual role of astrocyte activation and reactive gliosis. Neurosci Lett 565: 30-38.

40. Ridet JL, Malhotra SK, Privat A, Gage FH (1997) Reactive astrocytes: cellular and molecular cues to biological function. Trends Neurosci 20: 570-577.

41. Rosenfeld JV, McFarlane AC, Bragge P, Armonda RA, Grimes JB, Ling GS (2013) Blast-related traumatic brain injury. Lancet Neurol 12: 882-893.

42. Ryu J, Horkayne-Szakaly I, Xu L, Pletnikova O, Leri F, Eberhart C, Troncoso JC, Koliatsos VE (2014) The problem of axonal injury in the brains of veterans with histories of blast exposure. Acta Neuropathol Commun 2: 153.

43. Shitaka Y, Tran HT, Bennett RE, Sanchez L, Levy MA, Dikranian K, Brody DL (2011) Repetitive ClosedSkull Traumatic Brain Injury in Mice Causes Persistent Multifocal Axonal Injury and Microglial Reactivity. Journal of Neuropathology and Experimental Neurology 70: 551-567.

44. Siopi E, Calabria S, Plotkine M, Marchand-Leroux C, Jafarian-Tehrani M (2012) Minocycline restores olfactory bulb volume and olfactory behavior after traumatic brain injury in mice. J Neurotrauma 29: 354-361.

45. Sofroniew MV (2005) Reactive astrocytes in neural repair and protection. Neuroscientist 11: 400-407.

46. Stence N, Waite M, Dailey ME (2001) Dynamics of microglial activation: a confocal time-lapse analysis in hippocampal slices. GLIA 33: 256-266.

47. Suzumura A, Marunouchi T, Yamamoto H (1991) Morphological transformation of microglia in vitro. Brain Res 545: 301-306.

48. Tajiri N, Acosta SA, Shahaduzzaman M, Ishikawa H, Shinozuka K, Pabon M, Hernandez-Ontiveros D, Kim DW, Metcalf C, Staples M, Dailey T, Vasconcellos J, Franyuti G, Gould L, Patel N, Cooper D, Kaneko Y, Borlongan CV, Bickford PC (2014) Intravenous transplants of human adipose-derived stem cell protect the brain from traumatic brain injury-induced neurodegeneration and motor and cognitive impairments: cell graft biodistribution and soluble factors in young and aged rats. J Neurosci 34: 313326.

49. Xu L, Nguyen JV, Lehar M, Menon A, Rha E, Arena J, Ryu J, Marsh-Armstrong N, Marmarou CR, Koliatsos VE (2014) Repetitive mild traumatic brain injury with impact acceleration in the mouse: Multifocal axonopathy, neuroinflammation, and neurodegeneration in the visual system. Exp Neurol Epub ahead of print. 
50. Xu L, Ryu J, Nguyen JV, Arena J, Rha E, Vranis P, Hitt D, Marsh-Armstrong N, Koliatsos VE (2015) Evidence for accelerated tauopathy in the retina of transgenic P301S tau mice exposed to repetitive mild traumatic brain injury. Exp Neurol 273: 168-176.

51. Yamada J, Jinno S (2013) Novel objective classification of reactive microglia following hypoglossal axotomy using hierarchical cluster analysis. J Comp Neurol 521: 1184-1201.

52. Yan J, Xu L, Welsh AM, Hatfield G, Hazel T, Johe K, Koliatsos VE (2007) Extensive Neuronal Differentiation of Human Neural Stem Cell Grafts in Adult Rat Spinal Cord. PLoS Med 4: e39. 
Figure 1

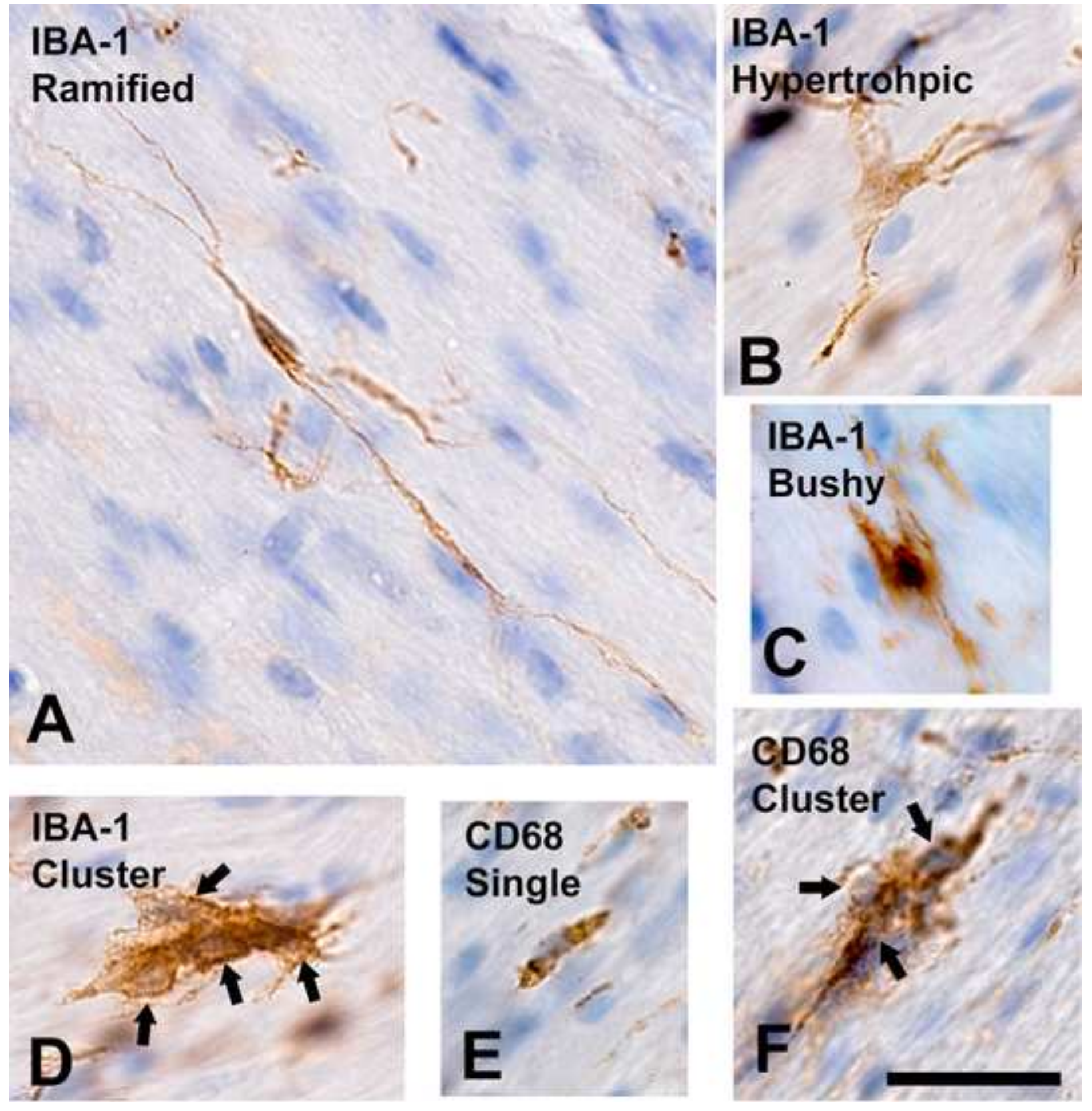



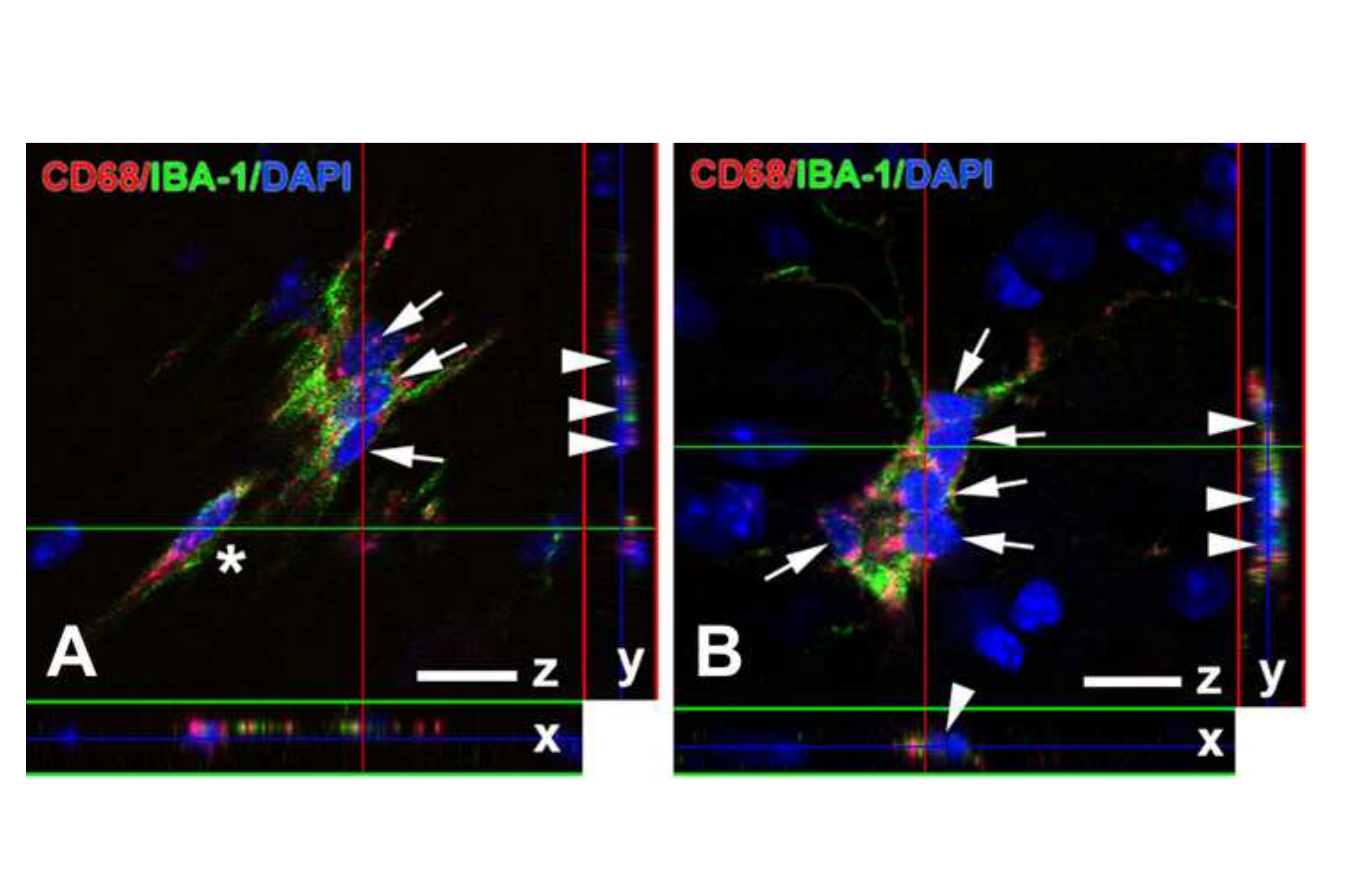

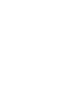

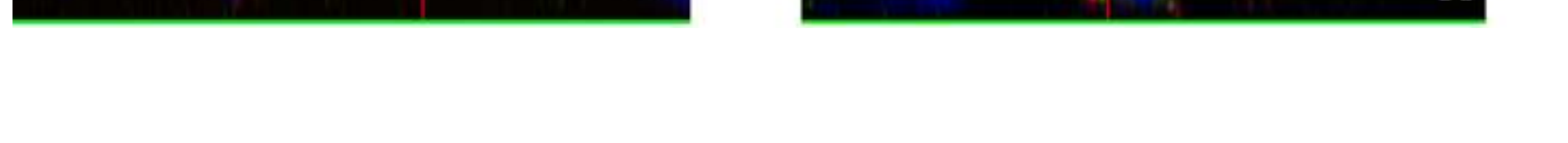

.

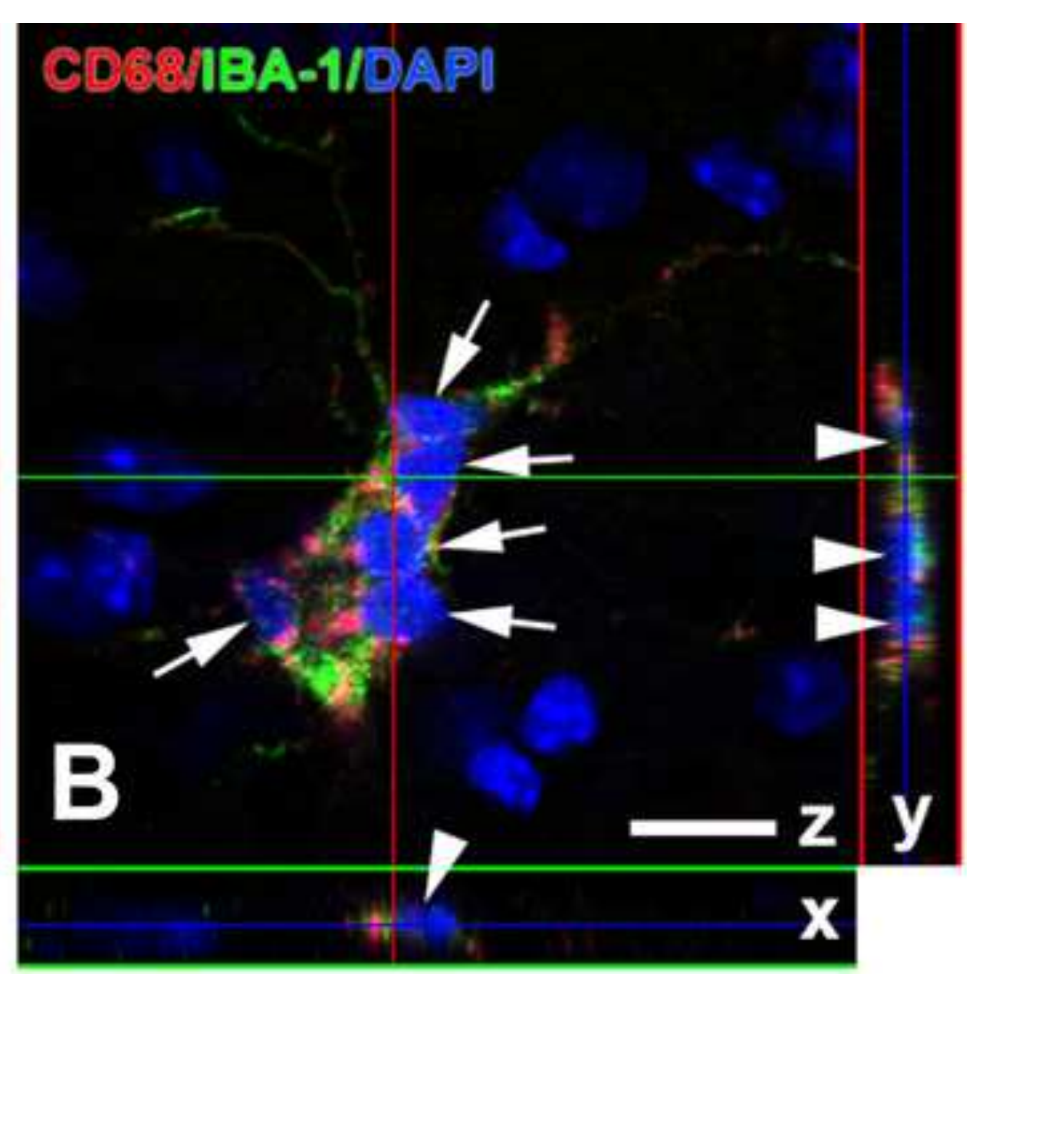




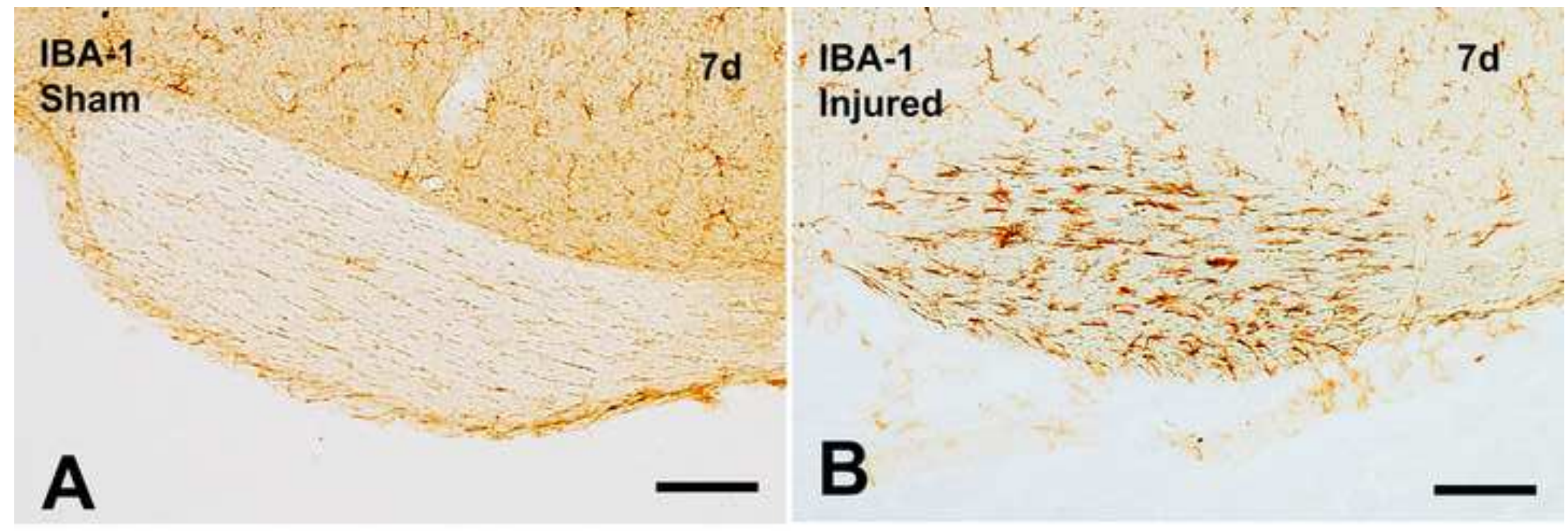

Activated IBA-1 (+) microglial profiles in optic tract

Total IBA-1 (+) microglia profiles in optic tract
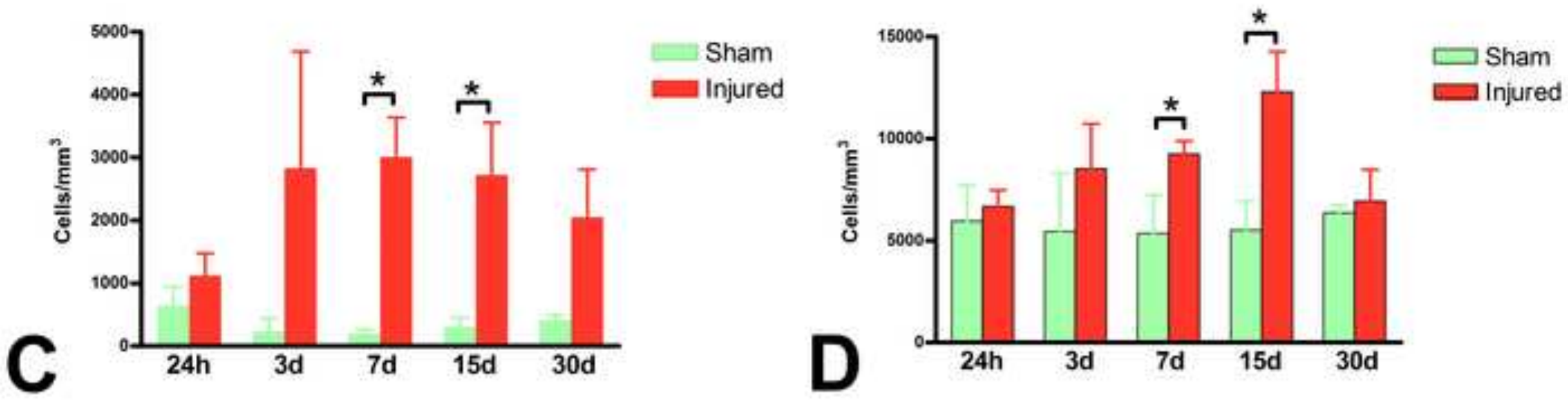
CD68 Sham

A

Single CD68 (+) microglial cells in optic tract

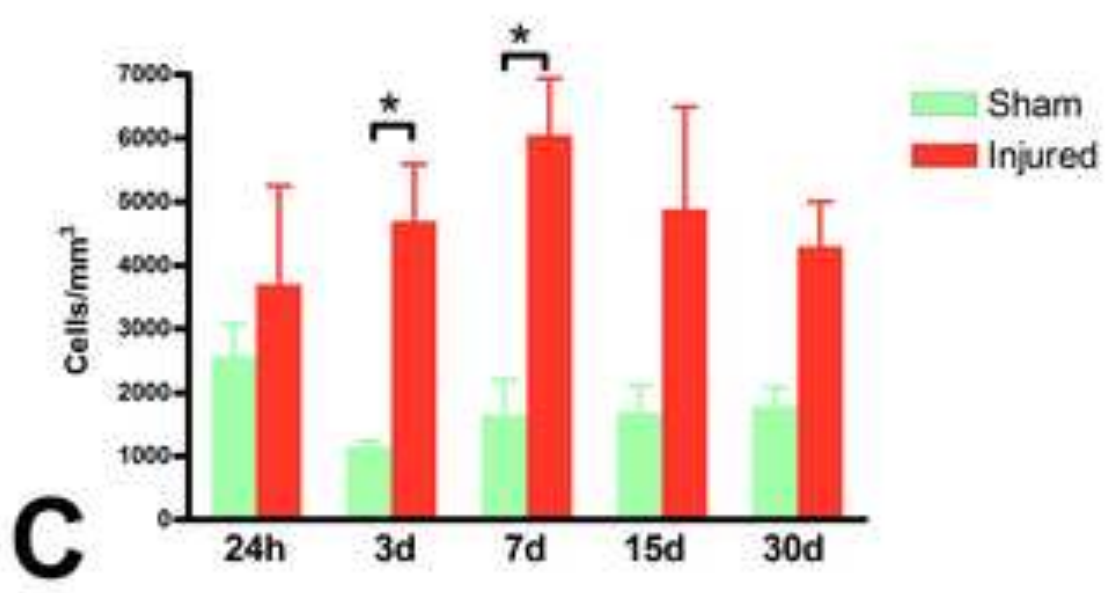

7d $\quad$ CD68 Injured

B

Clusters of CD68 (+) microglial cells in optic tract

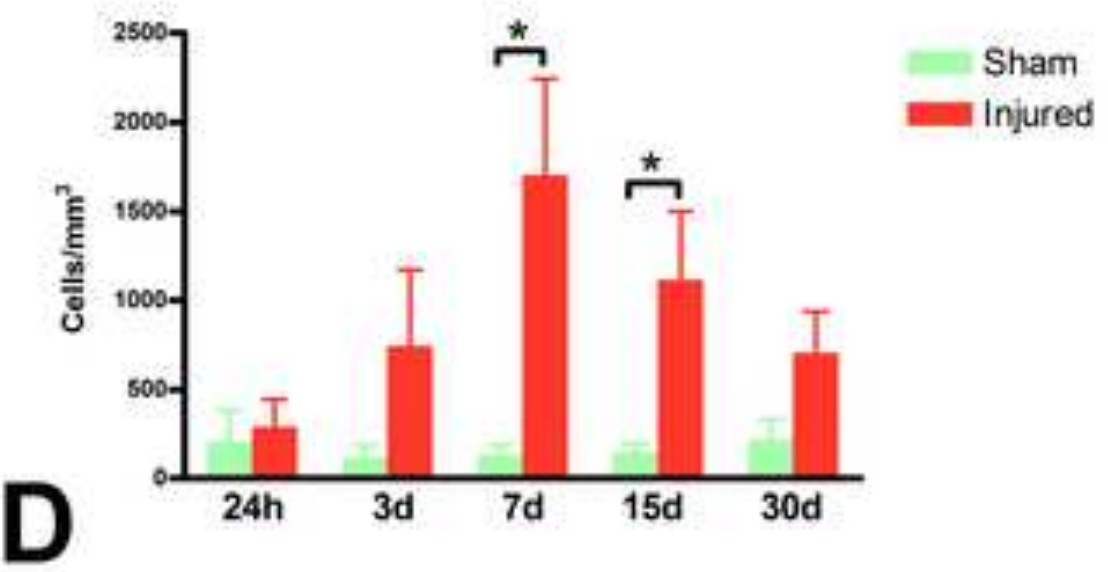


Activated IBA-1 (+) microglial profiles in optic tract

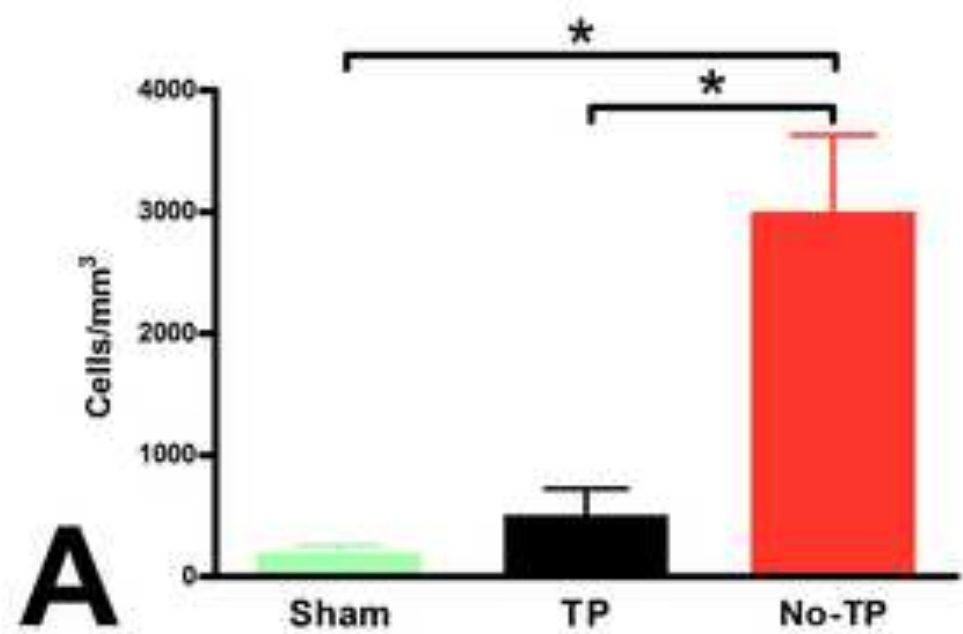

Single CD68 (+) microglial cells in optic tract

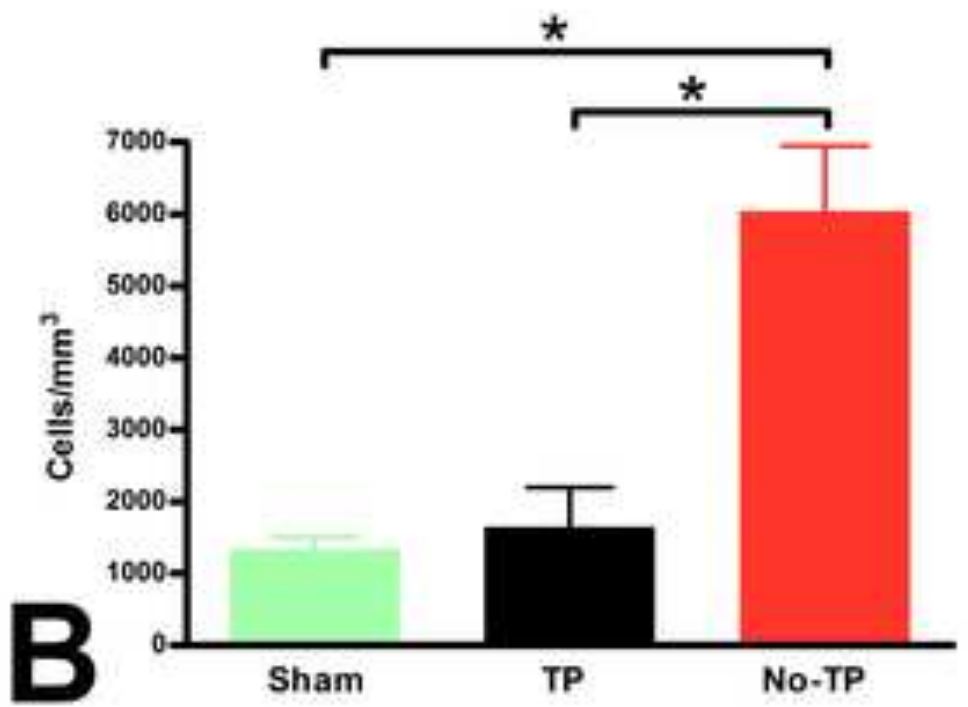

Clusters of CD68(+) microglial cells in optic tract

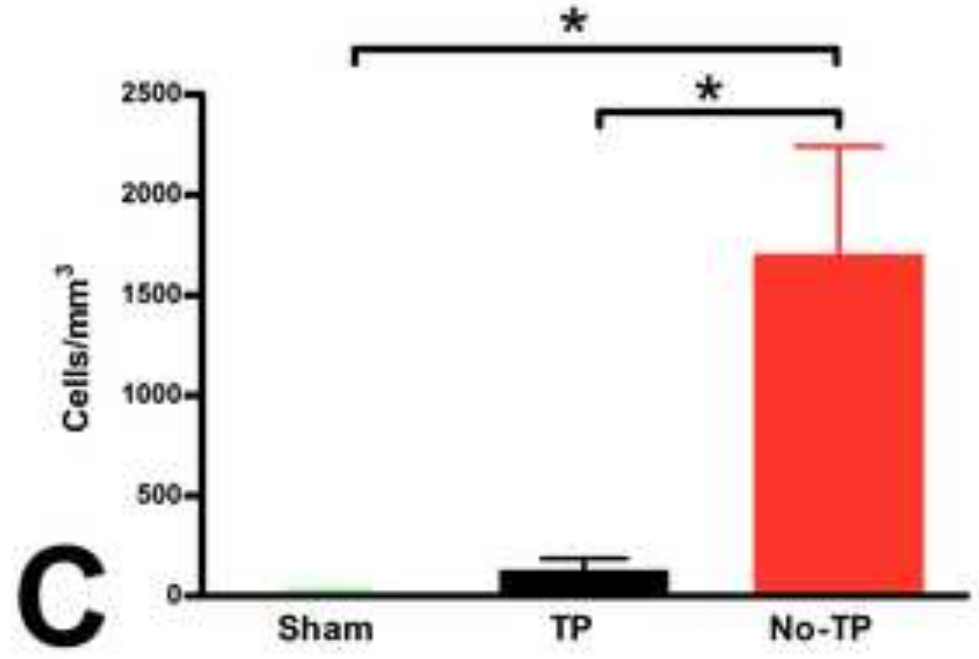

